\title{
Connected and disconnected quark contributions to hadron spin
}

\section{A. J. Chambers ${ }^{a}$, R. Horsley ${ }^{b}$, Y. Nakamura ${ }^{c}$, H. Perlt ${ }^{d}$, D. Pleiter ${ }^{e f}$, P.E.L. Rakow ${ }^{g}$, G. Schierholz ${ }^{h}$, A. Schiller ${ }^{c}$, H. Stüben ${ }^{i}$, R. D. Young ${ }^{a}$ and J. M. Zanotti ${ }^{* a}$}

${ }^{a}$ CSSM, Department of Physics, University of Adelaide, Adelaide SA 5005, Australia

${ }^{b}$ School of Physics and Astronomy, University of Edinburgh, Edinburgh EH9 3JZ, UK

${ }^{c}$ RIKEN Advanced Institute for Computational Science, Kobe, Hyogo 650-0047, Japan

${ }^{d}$ Institut für Theoretische Physik, Universität Leipzig, 04103 Leipzig, Germany

e JSC, Jülich Research Centre, 52425 Jülich, Germany

${ }^{f}$ Institut für Theoretische Physik, Universität Regensburg, 93040 Regensburg, Germany

${ }^{g}$ Theoretical Physics Division, Department of Mathematical Scienc University of Liverpool, Liverpool L69 3BX, UK

${ }^{h}$ Deutsches Elektronen-Synchrotron DESY, 22603 Hamburg, Germany

${ }^{i}$ Regionales Rechenzentrum, Universität Hamburg, 20146 Hamburg, Germany

E-mail: alexander.chambers@adelaide.edu.au,

james.zanotti@adelaide.edu.au

\begin{abstract}
By introducing an external spin operator to the fermion action, the quark spin fractions of hadrons are determined from the linear response of the hadron energies using the Feynman-Hellmann (FH) theorem. At our SU(3)-flavour symmetric point, we find that the connected quark spin fractions are universally in the range 55-70\% for vector mesons and octet and decuplet baryons. There is an indication that the amount of spin suppression is quite sensitive to the strength of SU(3) breaking. We also present first preliminary results applying the FH technique to calculations of quark-line disconnected contributions to hadronic matrix elements of axial and tensor operators. At the $\mathrm{SU}(3)$-flavour symmetric point we find a small negative contribution to the nucleon spin from disconnected quark diagrams, while the corresponding tensor matrix elements are consistent with zero.
\end{abstract}

The 32nd International Symposium on Lattice Field Theory,

23-28 June, 2014

Columbia University New York, NY

\footnotetext{
* Speaker.
} 


\section{Introduction}

Ever since the announcement by the European Muon Collaboration (EMC) [1] that quarks carry only a small fraction of the proton's spin, identifying the origin of hadronic spin has proven to be a fascinating challenge (see e.g. [2]). Since this is an inherently nonperturbative phenomenon, lattice QCD provides the ideal framework for investigating the spin decompostion of hadrons (see [3] for a review presented at this conference).

Standard methods for performing such a lattice QCD simulation involve calculating hadronic three-point functions via sequential source methods [4]. There has been increasing discussion surrounding the need for controlling potential excited state contamination in these three-point simulations, in particular for spin related quantities, such as $g_{A}$ [5-9]. Further challenges present themselves when calculting the contributions from quark-line disconnected diagrams (e.g. $\Delta s$ ), as these require the determination of computationally demanding all-to-all propagators. This problem is usually confronted through the use of stochastic methods, and there has been a lot of recent progress in this direction [10-14].

Recently we have proposed an alternative method for tackling these issues through the application of the Feynman-Hellmann (FH) theorem to lattice QCD calculations of hadronic matrix elements $[15,16]$, in a similar way to that proposed in [18]. We have also recently shown how it is possible to compute flavour-singlet renormalisation constants nonperturbatively by an appropriate application of the FH theorem [19].

In this talk, we present an update of the quark-line connected contributions to the spin of various hadrons first published in [16]. We will also reveal first simulations of the quark-line disconnected spin contributions through the generation of a new set of background gauge field configurations including the axial operator in the sea-quark action.

\section{The Feynman-Hellmann Theorem}

The Feynman-Hellmann theorem allows hadronic matrix elements to be calculated from shifts in the hadron spectrum. In general, if the action of our theory depends on some parameter $\lambda$, then for any hadron state $H$ we have

$$
\frac{\partial E_{H}}{\partial \lambda}=\frac{1}{2 E_{H}}\left\langle H\left|\frac{\partial S}{\partial \lambda}\right| H\right\rangle_{\lambda}
$$

where we use a subscript to indicate the $\lambda$ dependence of the matrix element on the right-hand side of Eq. 2.1. In particular, if we modify the QCD action such that

$$
S \rightarrow S^{\prime}(\lambda)=S+\lambda \int \mathrm{d}^{4} x O(x)
$$

where $O$ is some operator, then

$$
\left.\frac{\partial E_{H}}{\partial \lambda}\right|_{\lambda=0}=\frac{1}{2 E_{H}}\langle H|O| H\rangle
$$

noting the matrix element is now evaluated with respect to the unmodified action. 
The matrix element in Eq. 2.3 can be calculated by measuring shifts in the energy of the state $H$ as the parameter $\lambda$ is modified. A well-known example of this approach is the calculation of nucleon $\sigma$ terms, where the variational parameter(s) are the quark masses (see [17] for a review).

The Feynman-Hellmann method described can in general allow calculation of both connected and disconnected contributions to matrix elements. If the addition to the action in Eq. 2.2 is made during gauge field generation, one may make contact with the disconnected contributions, while modifications to the Dirac operator before calculating propagators allow access to the connected contributions.

\section{Lattice Details}

We use gauge field configurations with $2+1$ flavours of non-perturbatively $O(a)$-improved Wilson fermions and a lattice volume of $L^{3} \times T=32^{3} \times 64$. The lattice spacing $a=0.074(2) \mathrm{fm}$ is set using a number of singlet quantities [21-23]. The clover action used comprises the treelevel Symanzik improved gluon action together with a stout smeared fermion action, modified for the implementation of the Feynman-Hellmann method [16]. For the quark-line connected results, we use ensembles with three sets of hopping parameters, $\left(\kappa_{l}, \kappa_{s}\right)=(0.120900,120900)$, $(0.121040,120620),(0.121095,0.120512)$, corresponding to pion masses in the range $470-310$ $\mathrm{MeV}$.

The exploratory investigation of the quark-line disconnected contribution to the proton spin is performed at the $\mathrm{SU}(3)$ symmetric point $\left(\kappa_{l}=\kappa_{s}=0.120900\right)$ where all three quarks have the same mass $\left(m_{\pi} \approx 470 \mathrm{MeV}\right.$ ) and with two non-zero values of $\lambda$ applied equally to all three sea quarks.

In order to presently physically relevent results, we use recent nonperturbative determinations of the flavour non-singlet [24] and singlet [19] axial current renormalisation constants.

\section{Connected Spin Contributions}

To calculate connected contributions to the quark axial charges using the Feynman-Hellmann method, the fermion matrix for a single quark flavour is modified such that

$$
M \rightarrow M^{\prime}(\lambda)=M+\lambda i \gamma_{5} \gamma_{3} .
$$

where $i \gamma_{5} \gamma_{3}$ is the Euclidean form of the axial operator. Hence, for a general zero-momentum hadron state $H$, polarized in the $z$-direction, we have

$$
\left.\frac{\partial E_{H}}{\partial \lambda}\right|_{\lambda=0}=\frac{1}{2 M_{H}}\left\langle H ; J m\left|\bar{q} i \gamma_{5} \gamma_{3} q\right| H ; J m\right\rangle=\Delta q^{J m} .
$$

Here $J$ and $m$ are the spin and longitudinal spin polarisation quantum numbers, respectively. See [16] for a full discussion of this notation. Since the gauge fields used in this simulation were not generated with the modified operator in Eq. 4.1, we do not access disconnected contributions to the axial charges (as discussed in Sec. 2). We also note from Eq. 4.1 that reversing the spin of the hadron state is equivalent to flipping the sign of $\lambda$. So we may easily double our sampled parameter space by identifying, for example, spin-up nucleon states with positive $\lambda$, and spin-down states with negative $\lambda$. We improve the extracted signals by forming ratios of correlation functions 


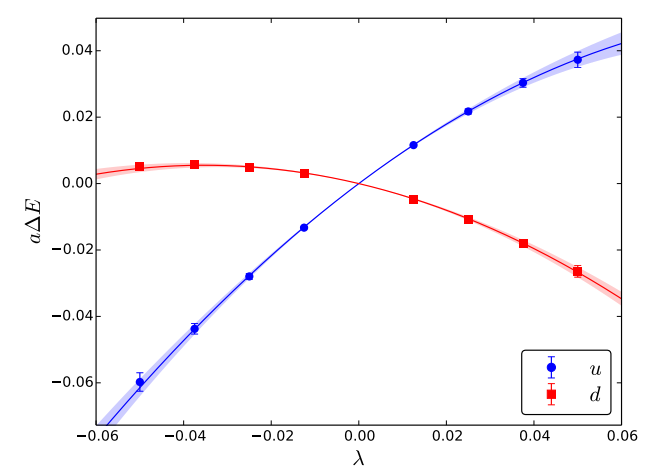

Figure 1: Nucleon energy shifts with respect to $\lambda$ at the $S U(3)$ symmetric point.

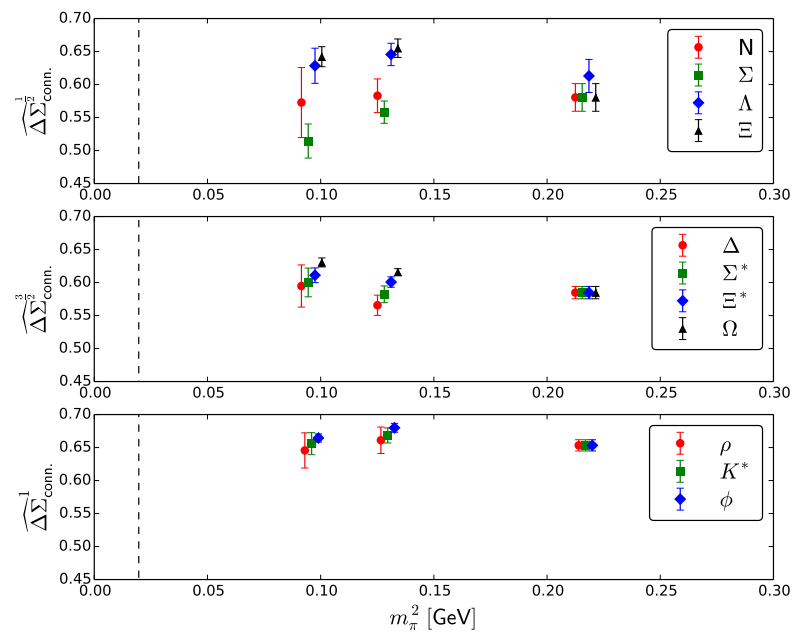

Figure 2: Total connected quark spin contributions to various hadrons as a function of pion mass.

at $\lambda \neq 0$ and $\lambda=0$, the details of which are discussed in [16]. Fig. 1 shows nucleon energy shifts for different values of $\lambda$, in the cases where the modification to the fermion matrix in Eq. 4.1 is made to the $u$ or $d$ part separately.

An important advantage of the Feynman-Hellmann method is that matrix elements of a particular operator may be easily calculated for different hadrons, without additional inversions being required. Fig. 2 shows results at three different pion masses for the total connected quark axial charges in various different hadrons.

Further discussion of these calculations may be found in [16].

\section{Disconnected Spin}

In principle, the application of the Feynman-Hellmann method to disconnected contributions is no less straightforward than the above, requiring only the modification of the fermion action during gauge field generation. However, an issue arises because the axial operator does not satisfy $\gamma_{5}$-hermiticity, and hence the modification to the Dirac operator in Eq. 4.1 generates a sign problem. To avoid this, we instead modify the fermion matrix such that

$$
M \rightarrow M^{\prime}(\lambda)=M+\lambda \gamma_{5} \gamma_{3}
$$

With this modification, the signal manifests as a complex phase in the correlation function

$$
C(\lambda, t)=A e^{-E t} e^{i \phi t},
$$

where any shift in $\phi$ with respect to $\lambda$ is related to the axial charge by

$$
\left.\frac{\partial \phi}{\partial \lambda}\right|_{\lambda=0}=\Delta q^{J m}
$$




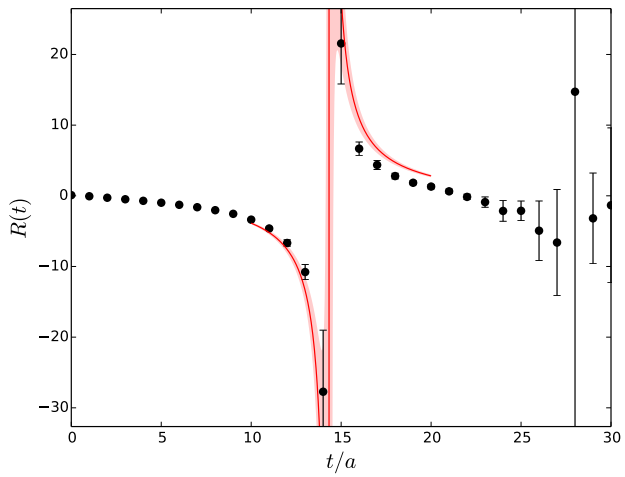

Figure 3: $R(\lambda, t)$ for the nucleon as defined in Eq. 5.4 for $\lambda_{\text {conn. }}=-0.1$, at the $\mathrm{SU}(3)$ symmetric point.

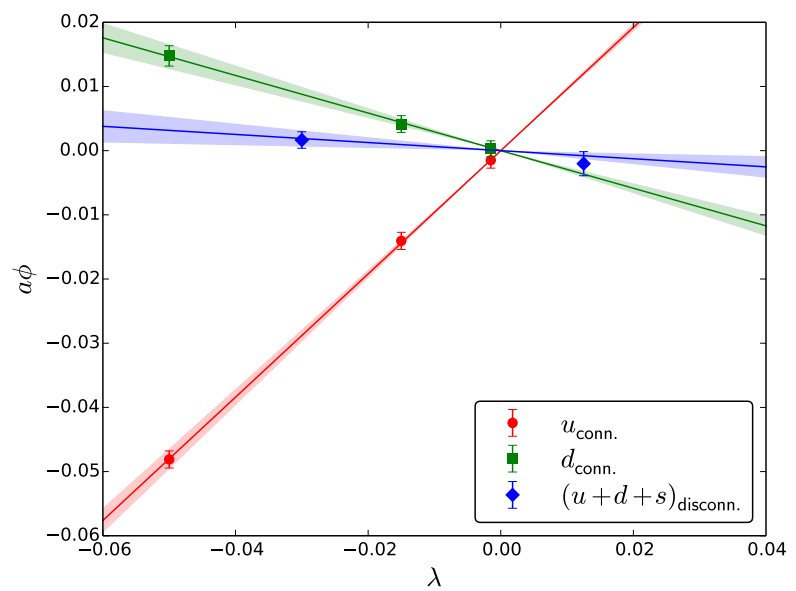

Figure 4: Complex phase of the nucleon correlator as a function of $\lambda$ at the $\mathrm{SU}(3)$ symmetric point. Connected and disconnected calculations are shown.

To first order in $\lambda$, the coefficient $A$ in Eq. 5.2 is real and there is no shift in $E$. This motivates the ratio

$$
R(\lambda, t)=\frac{\operatorname{Im} C(\lambda, t)-\operatorname{Im} C(-\lambda, t)}{\operatorname{Re} C(\lambda, t)+\operatorname{Re} C(-\lambda, t)}=\tan \phi t,
$$

where $C(-\lambda, t)$ is the spin-down hadron state at positive $\lambda$, recalling the discussion in Sec. 4 .

A plot of this ratio for a large value of $\lambda=-0.1$ can be seen in Fig. 3. With this background field strength, it is possible that terms beyond linear order in $\lambda$ influence the ratio, which would deviate from pure tangential behaviour. Nevertheless, we still observe the general tangent form. At the smaller $\lambda$ used for the Feynman-Hellmann calculation, this higher-order behaviour is not an issue.

Fig. 4 shows the change in the complex phase extracted from the tangential fit, with the background field strength. We are able to reproduce results for the connected contributions to the axial charges on the same ensemble (from [16], shown in brackets),

$$
\begin{array}{llrl}
\Delta u_{\text {conn. }}\left(m_{\pi} \approx 470 \mathrm{MeV}\right) & =0.816(33), & & {[0.849(17)]} \\
\Delta d_{\text {conn. }}\left(m_{\pi} \approx 470 \mathrm{MeV}\right)=-0.249(34) . & & {[-0.268(12)]}
\end{array}
$$

Using configurations generated with non-zero $\lambda$ applied to the sea quarks, we calculate from a linear fit to the blue data in Fig. 4, the total disconnected quark contribution to the axial charges,

$$
\Delta \Sigma_{\text {disconn. }}\left(m_{\pi} \approx 470 \mathrm{MeV}\right)=-0.055(36) .
$$

Here we make use of the singlet axial current renormalisation constant calculated in [19]. This is in agreement with stochastic estimations of this value reported in [11].

\section{Tensor Charge}

Calculation of the quark contributions to the tensor charge are calculated with the previously 


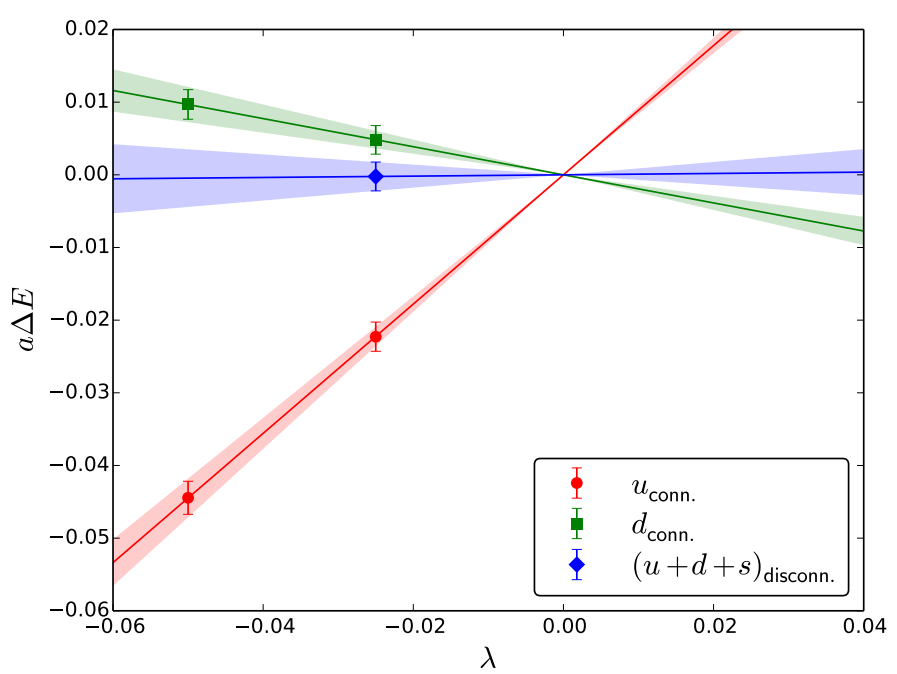

Figure 5: Nucleon energy shift with $\lambda$ when the modification described in Eq. 6.1 is made.

described methods. The fermion matrix is modified such that

$$
M \rightarrow M^{\prime}(\lambda)=M+\lambda \gamma_{5} \sigma_{34},
$$

so that the corresponding energy shifts are given by

$$
\left.\frac{\partial E_{H}}{\partial \lambda}\right|_{\lambda=0}=\frac{1}{2 M_{H}}\left\langle H ; J m\left|\bar{q} \gamma_{5} \sigma_{34} q\right| H ; J m\right\rangle
$$

We note that since the tensor operator flips helicity, the disconnected insertions of the operator must vanish in the chiral limit. However, away from the chiral limit, this is no longer guaranteed.

Fig. 5 shows nucleon energy shifts for the cases where the fermion matrix is modified before inversion (accessing the connected contributions to the tensor charge), and in the case where modified gauge fields are used to access the disconnected contributions. From a linear fit to the data, we calculate connected contributions

$$
\begin{aligned}
& \delta u_{\text {conn. }}\left(m_{\pi} \approx 470 \mathrm{MeV}\right)=0.881(17), \\
& \delta d_{\text {conn. }}\left(m_{\pi} \approx 470 \mathrm{MeV}\right)=-0.198(12),
\end{aligned}
$$

and disconnected contributions

$$
\delta(u+d+s)_{\text {disconn. }}\left(m_{\pi} \approx 470 \mathrm{MeV}\right)=-0.009(79),
$$

which is consistent with zero.

\section{Acknowledgments}

The numerical configuration generation was performed using the BQCD lattice QCD program, [25], on the IBM BlueGeneQ using DIRAC 2 resources (EPCC, Edinburgh, UK), the BlueGene P and Q at NIC (Jülich, Germany) and the Cray XC30 at HLRN (Berlin-Hannover, Germany). 
Some of the simulations were undertaken using resources awarded at the NCI National Facility in Canberra, Australia, and the iVEC facilities at the Pawsey Supercomputing Centre. These resources are provided through the National Computational Merit Allocation Scheme and the University of Adelaide Partner Share supported by the Australian Government. The BlueGene codes were optimised using Bagel [26]. The Chroma software library [27], was used in the data analysis. This investigation has been supported partly by the EU grants 283286 (HadronPhysics3), 227431 (Hadron Physics2) and 238353 (ITN STRONGnet), and by the Australian Research Council under grants FT120100821, FT100100005 and DP140103067 (RDY and JMZ).

\section{References}

[1] J. Ashman et al. [European Muon Collaboration], Phys. Lett. B 206 (1988) 364.

[2] A. W. Thomas, Int. J. Mod. Phys. E 18 (2009) 1116 [arXiv:0904.1735 [hep-ph]].

[3] M. Constantinou, PoS LATTICE 2014 (2014) 001 [arXiv:1411.0078 [hep-lat]].

[4] K. U. Can, A. Kusno, E. V. Mastropas and J. M. Zanotti, Lect. Notes Phys. 889 (2015) 69.

[5] B. J. Owen et al., Phys. Lett. B 723 (2013) 217 [arXiv:1212.4668 [hep-lat]].

[6] S. Capitani et al., Phys. Rev. D 86 (2012) 074502 [arXiv:1205.0180 [hep-lat]].

[7] S. Dinter et al., Phys. Lett. B 704 (2011) 89 [arXiv:1108.1076 [hep-lat]].

[8] T. Bhattacharya et al., Phys. Rev. D 89 (2014) 094502 [arXiv:1306.5435 [hep-lat]].

[9] G. S. Bali et al., PoS LATTICE 2013 (2014) 290 [arXiv:1311.7041 [hep-lat]].

[10] R. Babich et al., Phys. Rev. D 85 (2012) 054510 [arXiv:1012.0562 [hep-lat]].

[11] G. S. Bali et al., Phys. Rev. Lett. 108 (2012) 222001 [arXiv:1112.3354 [hep-lat]].

[12] M. Engelhardt, Phys. Rev. D 86 (2012) 114510 [arXiv:1210.0025 [hep-lat]].

[13] A. Abdel-Rehim et al., Phys. Rev. D 89 (2014) 3, 034501 [arXiv:1310.6339 [hep-lat]].

[14] M. Deka et al., arXiv:1312.4816 [hep-lat].

[15] R. Horsley et al., Phys. Lett. B 714 (2012) 312 [arXiv:1205.6410 [hep-lat]].

[16] A. J. Chambers et al., Phys. Rev. D 90 (2014) 014510 [arXiv:1405.3019 [hep-lat]].

[17] R. D. Young and A. W. Thomas, Nucl. Phys. A 844 (2010) 266C [arXiv:0911.1757 [hep-lat]].

[18] W. Detmold, Phys. Rev. D 71 (2005) 054506 [hep-lat/0410011].

[19] A. J. Chambers et al., Phys. Lett. B740 (2015) 30 [arXiv:1410.3078 [hep-lat]].

[20] N. Cundy et al., Phys. Rev. D79 (2009) 094507 [0901.3302 [hep-lat]].

[21] W. Bietenholz et al., Phys. Lett. B690 (2010) 436 [1003.1114 [hep-lat]].

[22] W. Bietenholz et al., Phys. Rev. D 84 (2011) 054509 [1102.5300 [hep-lat]].

[23] R. Horsley et al., PoS LATTICE 2013 (2014) 249 [arXiv:1311.5010 [hep-lat]].

[24] M. Constantinou et al., arXiv:1408.6047 [hep-lat].

[25] Y. Nakamura and H. Stüben, PoS LATTICE 2010 (2010) 040 [1011.0199 [hep-lat]].

[26] P. A. Boyle, Comput. Phys. Commun. 180 (2009) 2739.

[27] R. G. Edwards and B. Joó, Nucl. Phys. Proc. Suppl. 140 (2005) 832 [hep-lat/0409003]. 